\title{
Advanced Methodologies for Fracture Analysis and Damage Tolerant Evaluation
}

\author{
NAGESH R IYER ${ }^{1, *}$, G S PALANI ${ }^{2}$ and A RAMACHANDRA MURTHY ${ }^{3}$ \\ ${ }^{1}$ Distinguished Emeritus Professor, Academy of Scientific and Innovative Research (AcSIR) and Former \\ Director, CSIR-Structural Engineering Research Centre (CSIR-SERC) \\ ${ }^{2}$ Chief Scientist, ${ }^{3}$ Principal Scientist; CSIR-SERC
}

(Received on 10 March 2016; Accepted on 20 March 2016)

\begin{abstract}
The objective of the paper is to present the advanced methodologies for fracture analysis and damage tolerant evaluation of metallic structural components for efficient and safe design of structural systems meeting the functional criteria. An efficient and novel Numerically Integrated-Modified Virtual Crack Closure Integral (NI-MVCCI) technique has been demonstrated for 4-noded bilinear, 8-noded (regular \& quarter-point), 9-noded Lagrangian and 12-noded cubic isoparametric family of finite elements. Another advanced numerical method, Extended Finite Element Method (XFEM) for fracture analysis of cracked stiffened panels has been discussed. Methodologies for crack growth and remaining life assessment of structural components under constant and variable amplitude loading have been presented. Brief description on residual strength evaluation has been provided. Numerical studies on fracture analysis to compute Strain Energy Release Rate (SERR), G and stress intensity factor (SIF) and remaining life assessment and residual strength evaluation have been conducted and the results are compared with the respective analytical/experimental results.
\end{abstract}

Keywords: Crack Closure; Linear Elastic Fracture Mechanics; Stress Intensity Factor; Strain Energy Release Rate; Damage Tolerant Design; Remaining Life; Residual Strength; Extended Finite Element Method

\section{Introduction}

Many real life structural components such as aerospace structural components, ship hull structures, off shore structural components are subjected to fatigue loading. These components contain internal defects such as cracks, inclusions, voids which are developed during manufacturing stage or at service stage. The main function of a stiffener is to improve the strength and stability of the structure and to slow down or arrest the growth of cracks in the component. Remaining life prediction of the cracked structural components in these structures is necessary for their in-service inspection \& scheduling, planning, repair, retrofitting, rehabilitation, requalification and health monitoring. It is highly recommended to use the damage tolerant design concepts for designing structural components. Damage tolerant design is based on the information about the effect of cracks on the residual strength/remaining life of the structure.Practically, for all the high strength materials employed in the construction of above structures/ components, damage tolerant analysis can be performed using linear elastic fracture mechanics (LEFM) principles, in which case, SIF is the influencing design parameter. In general, it is difficult to quantify SIF for most of the practical applications. For simple geometries, SIF can be calculated by using handbooks (Rooke and Cartwright 1976; Murakami, 1988). There is a need to evolve efficient methodologies for computation of SIF and to provide an integrated approach that would include fatigue crack growth models for remaining life prediction. During the last four decades, a great deal of research (Poe, 1971; Chu et al., 1982; Ghassem and Rich, 1933; Wen et al., 2000, 2003; Saves et al., 2001; Dexter and Pilarsk, 2002; Taheri et al., 2003; Mahmoud and Dexter, 2005; Rama Chandra Murthy et al., 2007;

*Author for Correspondence: E-mail: nageshiyer@acsir.res.in 
Liu et al., 2012; Hosseini et al., 2013) has been dedicated to the development of numerical/analytical methods for computation of SIF for stiffened and unstiffened plate panels subjected to uniaxial tensile stresses.

The field of fracture mechanics is interdisciplinary requiring information from nondestructive techniques (NDT) to determine the size of possible pre-existing cracks, mechanical testing to determine fracture toughness of the material and stress analysis to determine the relevant fracture parameters required for the crack to grow (Zheng, 2001; Schijve, 2003). Work on NDT and the determination of fracture toughness are primarily experimental in nature whereas numerical techniques are the most preferred for stress analysis. Output of the stress analysis of cracked structures is essentially used in postprocessing stage to evaluate the well-known fracture parameters (Vasudevan et al., 2001; Broek, 1982) such as (i) SIFs, (ii) SERR and (iii) J-integral, in the realm of LEFM.

Among the various numerical techniques used for stress analysis, the finite element method (FEM) has evolved over the past 25 years as the most powerful numerical technique for the solution of solid mechanics problems (Zienkiewicz and Taylor, 2000). In recent years attention has been focused to study the fatigue and fracture behavior of complex structural components using FEM. Computation of SERR and SIF is a post-processing approach to finite element analysis (FEA). Advances in finite element (FE) technology with high performance computing have significantly improved the field of fracture mechanics. Number of software have been developed for fatigue and fracture analysis of structural components based on FEM. Some of the popular public domain and commercial software used for conducting fatigue and fracture analysis are: ZENCRACK, pc-CRACK, AFGROW and NASGRO.

Modelling of moving discontinuities (cracks) in finite element framework is a difficult task because of the need to update the mesh at each step as the crack propagates. A very fine mesh is required near the vicinity of the crack and mesh need to be conforming to the sides of the crack. Also the singularity at the crack tip must be accurately represented (Tong et al. 1973) Another way of handling discontinuities such as cracks is to model the crack, which is independent of the mesh. XFEM is one of the advanced numerical methods for fracture analysis of structural components. XFEM can be used to add discontinuous enrichment functions to the original finite element approximation (Belytschko et al., 1999; Moes et al., 1999; Dolbow et al., 2000) through the partition of unity (Melenk and Babuska, 1996). Automatic crack propagation can be obtained by properly implementing XFEM algorithms.

This paper presents an overview of the advanced methodologies for fracture analysis and damage tolerant evaluation of structural components. The novel contributions presented in this paper include:

- $\quad$ NI-MVCCI technique for fracture analysis

- $\quad$ XFEM for stiffened panels

- Improved Wheeler residual stress model to account for retardation effects due to overload

- Residual strength evaluation using remaining life approach

\section{Formulation of NI-MVCCI Technique (Palani et al., 2004)}

The derivation of element dependent MVCCI expressions for computing SERR for mode I and II cracks $\left(\mathrm{G}_{\mathrm{I}}\right.$ and $\left.\mathrm{G}_{\mathrm{II}}\right)$ for 4-noded element, 8-noded element and 8-noded QPE involves evaluation of constants used in the polynomial assumed to represent displacement and stress variation and evaluation of many integrals having polynomial terms. As mentioned earlier, the derivation of MVCCI expressions becomes a tedious exercise for higher order 2-D (such as 12noded singular) and 3-D (such as HEXA20 and HEXA27) finite elements. One of the main objectives of NI-MVCCI technique is to overcome this tedious exercise by involving numerical techniques for computation of the constants and to evaluate CCI for $\mathrm{G}_{\mathrm{I}}$ and $\mathrm{G}_{\mathrm{II}}$. NI-MVCCI is generalized technique and is independent of the type of finite element employed. Consider a typical FE mesh at the crack tip as shown in Fig. 2. The procedure for evaluating MVCCI for mode I and II cracks in 2-D problems using numerical techniques is explained. For mode I crack, $\operatorname{SERR}\left(\mathrm{G}_{\mathrm{I}}\right)$ can be evaluated by multiplying the stress distribution along OA (ahead of crack tip) with the corresponding COD distribution along OB (behind crack tip) and integrating this product over Da. 


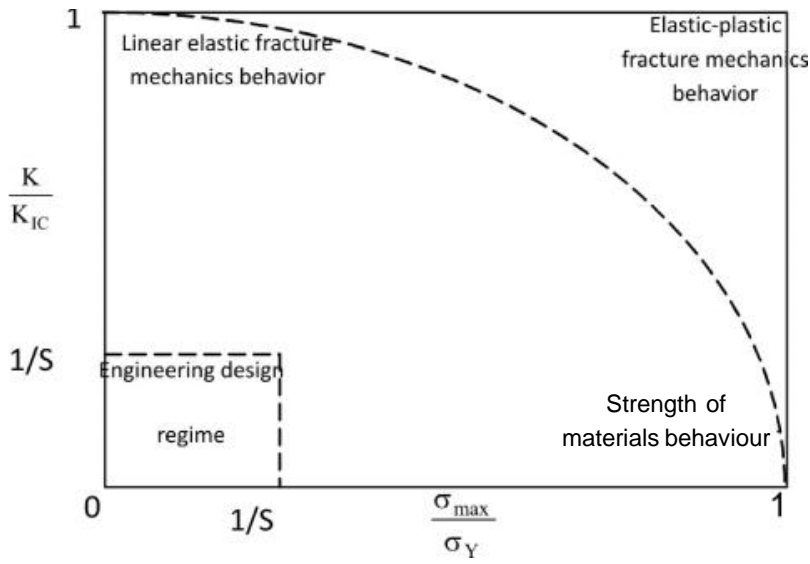

Fig. 1: Structural integrity assessment diagram

For evaluation of SERR for mode $I$ crack $\left(G_{I}\right)$ the stress distribution on the crack extension line OA is expressed in terms of the nodal forces $F_{y, j}, F_{y,(j+1)}$, etc. acting at the nodes $j,(j+1)$, etc. respectively. COD distribution along OB is expressed in terms of the nodal values at $j,(j-1),(j-1)$, etc. SERR for mode $I$ crack $\left(G_{I}\right)$ is derived by evaluating the energy required to close the crack over a length $\Delta \mathrm{a}$ in terms of these nodal forces and displacements. The shape functions for elements 1 and 2 along $\mathrm{OB}$ can be obtained by substituting $\eta=-1$, in the respective element shape functions. Let these shape functions be $N_{i}$. Let COD which is the difference between $y$ displacement between the top and bottom faces be designated as $U_{y}$ in the discussion in this section. Similarly crack shearing displacement be designated as $U_{x}$. COD distribution along $O B$ can be expressed in terms of nodal displacements $\left\{\left(U_{y}\right)_{i}\right\}$ as

$$
U_{y}=\left[N_{i}\right]\left\{\left(U_{y}\right)_{i}\right\} \quad i=1, \ldots . n
$$

where $n$ is the number of nodes on the edge OA or $\mathrm{OB}$ of the element. Consistent with the isoparametric formulation, coordinate of any point $X(x, y)$ is given by

$$
X=\left[N_{i}\right]\left\{(X)_{i}\right\} \quad i=1, \ldots . n
$$

where $\left\{(X)_{i}\right\}$ are the nodal coordinates. Eq. (2) thus provides the transformation between the global and natural coordinate system. Consistent with the element shape functions, COD variation along $\mathrm{OB}$ can be expressed as function of $\xi$ ' as

$$
U_{y}\left(\xi^{\prime}\right)=\mathrm{a}_{0}+\mathrm{a}_{1} \xi^{\prime}+\ldots . .+\mathrm{a}_{(n-1)} \xi^{\prime(n-1)}
$$

where $U_{y}\left(\xi^{\prime}\right)$ is a polynomial of order $(n-1)$. The constants $\mathrm{a}_{0}, \mathrm{a}_{1}, \ldots, \mathrm{a}_{(n-1)}$ can be evaluated by matching the displacement conditions at the nodes $j$, $(j-1), \ldots,(j-n+1)$ in element number 1 . A set of simultaneous equations of order $\mathrm{n}$ is formed, which can be solved for obtaining the constants $\mathrm{a}_{0}, \mathrm{a}_{1}, \ldots$, $\mathrm{a}_{(n-1)}$. Again referring to Fig. 5 and considering element number 2 , stress $\left(\sigma_{y}\right)$ distribution along OA can be expressed as a function of $\xi$

$$
\sigma_{y}(\xi)=b_{0}+b_{1} \xi+\ldots b_{(n-1)} \xi^{(n-1)}
$$

where $\sigma_{y}(\xi)$ is a polynomial of order $(n-1)$. The constants $\mathrm{b}_{0}, \mathrm{~b}_{1}, \ldots, \mathrm{b}_{(n-1)}$ can be computed by matching the nodal forces with the derived consistent load vector from FE analysis. The nodal forces $F_{y, j}$, $F_{y,(j+1)}, \ldots, F_{y,(j+n-1)}$ shown in Fig. 2 are the forces exerted at nodes $j,(j+1), \ldots,(j+n-1)$ by the structure below OA on the structure above OA. In FEA, these forces are obtained by adding the contributions of the forces at nodes $j,(j+1), \ldots,(j+n-1)$ from the elements on the edge above OA. These forces should be consistent with the stress distribution given in eqn (4), which can be expressed as

$$
F_{i}=\int_{\Delta \mathrm{a}}\left[N_{i}\right]^{T} \sigma_{y}(\xi) d x \quad i=1, . n
$$

where $N_{i}$ are the shape functions of the respective element obtained by substituting $\eta=-1$. By using the transformation between the global and natural coordinate system (eqn (2)), the basis can be shifted from ' $\mathrm{dx}$ ' to ' $\mathrm{d} \xi$ '. The integrals given in eqn (12) can be evaluated by numerical integration technique with suitable order. In the present study, Gauss integration technique has been employed with different integration rules. By substituting the expressions for COD and $\sigma_{y}$ stress variations given by eqns (3) and (4) respectively in CCI, $G_{I}$ can be expressed as

$$
G_{I}=\underset{\Delta \mathrm{a} \rightarrow 0}{\operatorname{Lt}} \frac{1}{2 \Delta \mathrm{a}} \int_{\Delta \mathrm{a}} \sigma_{y}(\xi) U_{y}\left(\xi^{\prime}\right) d x
$$

Similarly crack shearing displacement $U_{x}\left(x^{\prime}\right)$ and $\sigma_{x y}$ distributions can be developed and $\mathrm{G}_{\mathrm{II}}$ can be expressed as

$$
G_{I I}=\underset{\Delta \mathrm{a} \rightarrow 0}{\operatorname{Lt}} \frac{1}{2 \Delta \mathrm{a}} \int_{\Delta \mathrm{a}} \sigma_{x y}(\xi) U_{x}\left(\xi^{\prime}\right) d x
$$


The integrals given in Eqs. (6) and (7) can be performed by numerical integration technique. The numerical integration has to be carried out in two stages: One for evaluating the constants in $\sigma_{y}\left(\right.$ or $\left.\sigma_{x y}\right)$ distributions in terms of the nodal forces $F_{y}\left(\right.$ or $\left.F_{x}\right)$ in Eq. (5) and the second to evaluate the integral for $\mathrm{G}_{\mathrm{I}}$ (or $\mathrm{G}_{\mathrm{II}}$ ) in eqn (6) (or eqn (7). In the present study, Gauss integration technique has been employed in eqn (6) with the same integration rule that is used for evaluating Eq. (5).

It can be observed from Eqs. (1) to (7), the proposed NI-MVCCI is a generalized technique and is independent of the type of element, except for assuming the expressions for displacement and stress variation (Eqs. (3) and (4)). The order of polynomials for displacement and stress variation can be assumed to be consistent with the element shape functions along the edge $\eta=-1$. NI-MVCCI technique explained above can be easily implemented in any finite element code which has 4-noded, 8-noded (regular and quarterpoint), 9-noded (regular and quarter-point) and 12noded (regular \& singular) quadrilateral isoparametric elements. However, some basic discussion on the polynomials to be selected will be required for implementation of NI-MVCCI technique for these elements. These basic aspects for each of these elements corresponding to Eqs. (8)-(14) are now presented.

\section{8-noded and 9-noded (regular) Quadrilateral Elements}

Shape functions $\left(N_{i}\right)$ in eqns (1) and (5) along the edge OB as shown in Fig. 2 (for the edge OA replace $\xi^{\prime}$ by $\xi$ ) can be obtained by substituting $\eta=-1$ in 8 noded or 9-noded element shape functions

$$
N_{i}=1 / 2\left(1+\xi^{\prime} \xi_{i}^{\prime}\right) \xi^{\prime} \xi_{i}^{\prime} \text { for nodes with }
$$

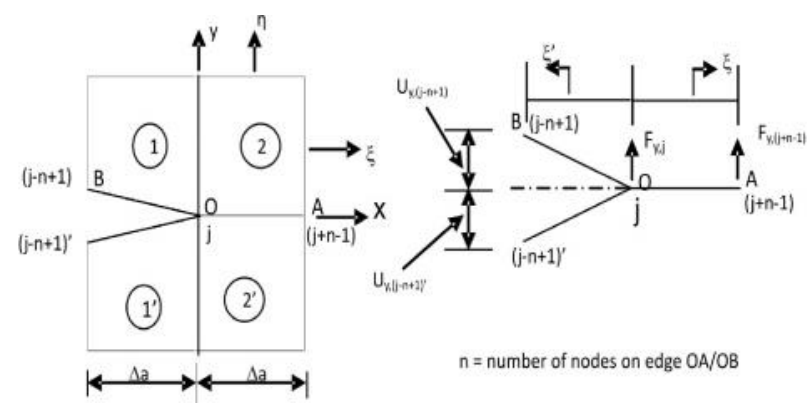

Fig. 2: Typical FE mesh of crack tip region

$$
\begin{aligned}
\xi^{\prime} & = \pm 1 \\
& =1 / 2\left(1-\xi^{\prime}\right) \text { for node with } \xi^{\prime}=0
\end{aligned}
$$

Using the shape functions given in Eq. (8a), the transformation between global and natural coordinate system as given in Eq. (5) can be expressed as

$$
x=-(\Delta \mathrm{a} / 2)\left(1+\zeta^{\prime}\right)
$$

By substituting $n=3$ in Eq. (3), COD variation along OB can be assumed as

$$
U_{y}\left(\xi^{\prime}\right)=a_{0}+a_{1} \xi^{\prime}+a_{2} \xi^{\prime 2}
$$

By substituting $n=3$ in Eq. (4), the stress variation along $\mathrm{OA}$ can be assumed as

$$
\sigma_{y y}(\xi)=\mathrm{b}_{0}+\mathrm{b}_{1} \xi+\mathrm{b}_{2} \xi^{2}
$$

Displacement and force conditions for evaluating the constants $a_{0}, a_{1}, a_{2}$ and $b_{0}, b_{1}, b_{2}$ can be expressed as

$$
\begin{aligned}
& U_{y}=0 \text { at } \xi^{\prime}=-1 ; U_{y}=\left(U_{y,(j-1)}-U_{y,(j-1)}\right) \\
& \text { at } \xi^{\prime}=0 ; U_{y}=\left(U_{y,(j-2)}-U_{y,(j-2)},\right) \text { at } \xi^{\prime}=1 ; \\
& F_{y}=F_{y, j} \text { at } \xi=-1 ; F_{y}=F_{y,(j+1)} \text { at } \xi=0 ; \\
& F_{y}=F_{y,(j+2)} \text { at } \xi=1 ;
\end{aligned}
$$

Referring to Fig. 2, the relation between $\xi^{\prime}$ in element 1 and $\xi$ in element 2 can be expressed as $\xi^{\prime}=-\xi$.

\section{8-noded and 9-noded QPE}

Shape functions $\left(N_{i}\right)$ in Eqs. (1) and (5) along the edge OB as shown in Fig. 2 (for the edge OA replace $\xi^{\prime}$ by $\xi$ ) can be obtained by substituting $\eta=-1$ in 8 noded QPE shape functions

$$
\begin{aligned}
& N_{i}=1 / 2\left(1+\xi^{\prime} \xi_{i}^{\prime}\right) \xi^{\prime} \xi_{i}^{\prime} \text { for nodes with } \\
& \xi^{\prime}= \pm 1 \\
& N_{i}=1 / 2\left(1-\xi^{\prime 2}\right) \text { for node with } \xi^{\prime}=0
\end{aligned}
$$

Using the shape functions given in Eq. (9a), the transformation between global and natural coordinate system as given in Eq. (2) can be expressed as 


$$
x=(\Delta \mathrm{a} / 4)\left(1+\xi^{\prime}\right)^{2}
$$

By substituting $n=3$ in Eq. (3) and accounting for the quarter-point position of the mid-side node, COD variation along OB can be assumed as

$$
U_{y}\left(\xi^{\prime}\right)=\mathrm{a}_{0}+\mathrm{a}_{1}\left(1+\xi^{\prime}\right)+\mathrm{a}_{2}\left(1+\xi^{\prime}\right)^{2}
$$

In order to account for the singular stress conditions represented by QPE, the stress variation along OA can be assumed as

$$
\sigma_{y y}(\xi)=\mathrm{b}_{0} /(1+\xi)+\mathrm{b}_{1}+\mathrm{b}_{2}(1+\xi)
$$

Displacement and force conditions for evaluating the constants $\mathrm{a}_{0}, \mathrm{a}_{1}, \mathrm{a}_{2}$ and $\mathrm{b}_{0}, \mathrm{~b}_{1}, \mathrm{~b}_{2}$ can be expressed as

$$
\begin{aligned}
& U_{y}=0 \text { at } \xi^{\prime}=-1 ; \quad U_{y}=\left(U_{y,(j-1)}-U_{y,(j-1)^{\prime}}\right) \\
& \text { at } \xi^{\prime}=0 ; U_{y}=\left(U_{y,(j-2)}-U_{y,(j-2)}\right) \\
& \text { at } \xi^{\prime}=1 \\
& F_{y}=F_{y, j} \text { at } \xi=-1 ; F_{y}=F_{y,(j+1)} \text { at } \xi=0 \\
& F_{y}=F_{y,(j+2)} \text { at } \xi=1 ;
\end{aligned}
$$

Referring to Fig. 2 and accounting for the quarter-point position of the mid-side node, the relation between $\xi^{\prime}$ in element 1 and $\xi$ in element 2 can be expressed as

$$
(1+\xi)^{2}+\left(1+\xi^{\prime}\right)^{2}=4
$$

\section{XFEM for Fracture Analysis and Remaining Life Prediction}

In XFEM, the following approximation is used to compute the displacement for the point $\mathbf{x}$ located within the domain (Belytschko et al., 1999)

$$
\begin{aligned}
\mathbf{u}^{h}(\mathbf{x}) & =\mathbf{u}^{F E}+\mathbf{u}^{e n r} \\
& =\sum_{i=1}^{n} N_{i}(\mathbf{x}) \mathbf{u}_{\mathbf{i}}+\sum_{k=1}^{m} N_{k}(\mathbf{x}) \psi(\mathbf{x}) \mathbf{a}_{\mathbf{k}}
\end{aligned}
$$

where $\mathbf{u}_{\mathrm{i}}$ is the vector of regular nodal degrees of freedom (DOF) in FEM, $\mathrm{n}$ is the total number of nodes in FE model, $\mathrm{N}_{\mathrm{i}}$ shape function associated with node $\mathrm{i},{ }_{\mathrm{ak}}$ is the added set of DOF to the standard FE model, $\mathrm{m}$ is the number of enriched nodes and $\psi(\mathbf{x})$ is the discontinuous enrichment function defined for the set of nodes that the discontinuity has in its influence (support) domain. The enrichment function $\psi(\mathbf{x})$ can be chosen by applying appropriate analytical solutions according to the type of discontinuity. The first term on the right hand side of Eq. (10) is the classical FE approximation to determine the displacement field, while the second term is the enrichment approximation, which takes into account the existence of any discontinuities. The second term utilises additional degrees of freedom to facilitate modelling the existence of any discontinuous field, such as a crack, without modelling it explicitly in the finite element mesh.

When XFEM is applied to fracture mechanics problems, displacement field is taken as (Moes et al. 1999).

$$
\begin{aligned}
& \mathbf{u}^{h}(\mathbf{x})=\sum_{i=1}^{n} N_{i}(\mathbf{x}) \mathbf{u}_{\mathbf{i}}+\sum_{j \in J} N_{j}(\mathbf{x}) H(\mathbf{x}) \mathbf{a}_{\mathbf{j}} \\
& +\sum_{k \in K_{1}} N_{k}\left(\sum_{l=1}^{4} F_{l}^{1}(\mathbf{x}) \mathbf{b}_{\mathbf{k l}}^{\mathbf{1}}\right) \sum_{k \in K_{2}} N_{k}\left(\sum_{l=1}^{4} F_{l}^{2}(\mathbf{x}) \mathbf{b}_{\mathbf{k l}}^{\mathbf{2}}\right)
\end{aligned}
$$

where, $H(x)$ is the heaviside enrichment function defined such that it equals 1 for all $\mathbf{x}$ above the crack and -1 for all $\mathbf{x}$ below the crack as shown in Fig. 3 and $\mathbf{a}_{\mathbf{j}}$ is the heaviside enriched node. $\mathbf{J}$ is the set of nodes, enriched with heaviside enrichment function, whose nodal shape function support contain crack but not crack tip.

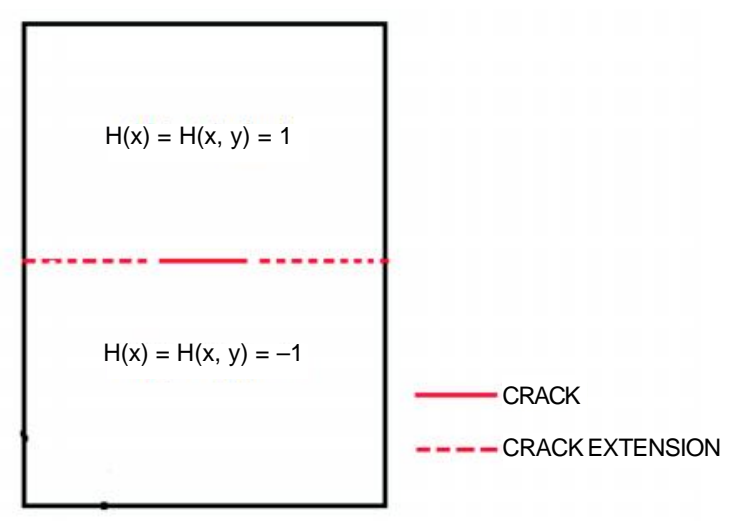

Fig. 3: Heaviside enrichment function definition 
Here $\mathrm{k}_{1}$ and $\mathrm{k}_{2}$ are the set of nodes, associated with crack tips 1 and 2 , whose element contain crack tips respectively; $\mathrm{b}_{k l}^{1}, \mathrm{~b}_{k l}^{2}$, are vectors of additional nodal DOF for modelling crack faces and the two crack tips.

Typical flow chart for fracture analysis and remaining life prediction is given in Fig. 4.

\section{Modelling of Stiffeners in XFEM Domain}

As the stiffeners are placed concentric and the applied loading is tensile in nature, one can model the stiffeners using truss elements. It is assumed that stiffeners are continuous and are connected to the plate along the nodes of the plate element modelled as bilinear

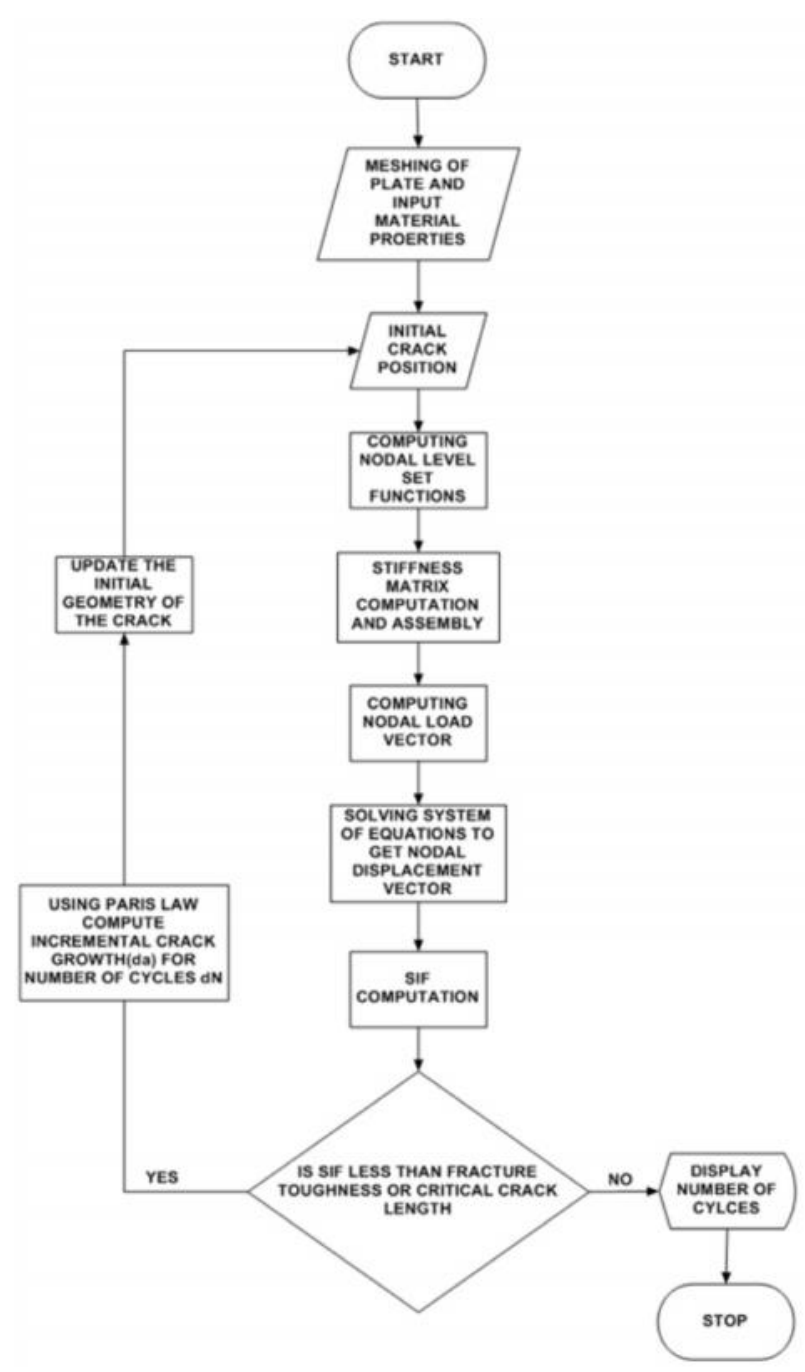

Fig. 4: Typical XFEM flow chart for fracture analysis and remaining life prediction element. For example consider a square plate of side L modelled using four bilinear elements of side 1 and an edge stiffener using two node truss element as shown in Fig. 5 (Nanda Kumar et al. 2016).

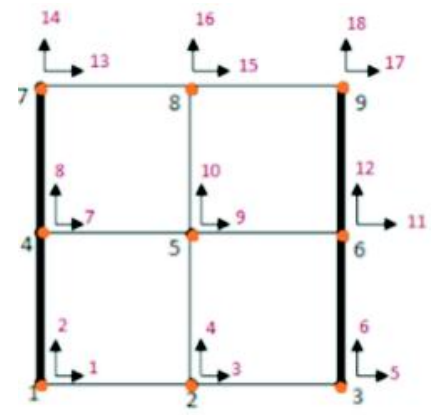

(A)

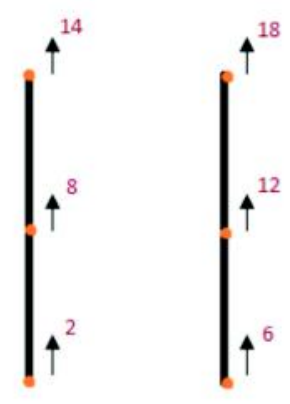

(B)
Fig. 5: (A) Finite element model of plate using four node bilinear element and (B) Finite element model of stiffeners using two node truss element

Assuming that the Young's modulus of stiffener and Young's modulus of panel is same and denoted it as E and area of cross section of each stiffener as A. The length of truss element is same as side of bilinear element. Thus element stiffness matrix of truss element is given as

$$
k=\frac{\mathrm{AE}}{1}\left[\begin{array}{cc}
1 & -1 \\
-1 & 1
\end{array}\right]
$$

The global stiffness matrix of panel modelled using four node bilinear element be $K_{p}$. Size of $K_{p}$ depends upon the location and position of the crack in the panel. The assembled stiffness matrix of stiffeners modelled as truss elements can be obtained as

$$
\begin{aligned}
K_{t 1} & =\frac{A E}{l}\left[\begin{array}{ccc}
1 & -1 & 0 \\
-1 & 2 & -1 \\
0 & -1 & 1
\end{array}\right]_{14}^{2} \\
K_{t 2} & =\frac{A E}{l}\left[\begin{array}{ccc}
1 & -1 & 0 \\
-1 & 2 & -1 \\
0 & -1 & 1
\end{array}\right] \begin{array}{l}
6 \\
12
\end{array}
\end{aligned}
$$

where $\mathrm{K}_{\mathrm{t} 1}$ and $\mathrm{K}_{\mathrm{t} 2}$ are the assembled global stiffness matrices of stiffener 1 and stiffener 2 respectively each of size equal to $K_{p}$. Note that the matrices shown 
in Eqs. (13) and (14) are only sub matrices and the values in other locations of matrices $\mathrm{K}_{\mathrm{t} 1}$ and $\mathrm{K}_{\mathrm{t} 2}$ are zero

Thus the final stiffness matrix of panel and stiffener is

$$
\mathrm{K}=\mathrm{K}_{\mathrm{p}}+\mathrm{K}_{\mathrm{t} 1}+\mathrm{K}_{\mathrm{t} 2}
$$

Similar lines, the final stiffness matrix of panel and stiffener for various mesh discretizations can be formulated.

\section{Damage Tolerant Evaluation}

Damage tolerant evaluation involves the techniques for arriving at the residual strength diagram and the crack growth diagram. From the residual strength diagram, it is possible to determine the maximum crack length that can be sustained safely. This information is used in the crack growth diagram to find number of loading cycles that will be necessary for the crack to grow to its critical length. So, obviously damage tolerant design requires reliable estimation of fatigue crack growth.

\section{Crack Growth Models}

The crack growth models address the crack growth behaviour by using SIF at crack tip. The amount of crack advancement, $\Delta \mathrm{a}=\mathrm{a}_{\mathrm{f}}-\mathrm{a}_{\mathrm{i}}$, during $\Delta \mathrm{N}$ number of cycles is described by SIF range associated with maximum and minimum stress of each cycle $\left(\mathrm{K}_{\max }-\right.$ $\mathrm{K}_{\text {min }}$ ). The rate of crack growth, da/dN, in terms of the crack tip SIF range, DK can be expressed as

$$
\frac{d \mathrm{a}}{d N}=f(\Delta K)
$$

The function $f(\Delta K)$ can be obtained as a result of experimental data and can then be utilized to solve crack growth problems in which the structural part has undergone the same loading conditions.

\section{Models for Fatigue Crack Growth under Constant Amplitude Loading (CAL)}

Paris formulated the earliest relationship describing crack growth behaviour. When a cracked structural joint or component is subjected to cyclic loading, the crack propagation rate, da/dN is governed by SIF range $\Delta \mathrm{K}$ as for a standard through thickness cracked specimen under constant amplitude fatigue loading,

$$
\frac{d \mathrm{a}}{d N}=C_{p}(\Delta K)^{m_{p}}
$$

where $\mathrm{N}$ is the number of cycles, $\mathrm{C}$ and $\mathrm{m}$ are constants dependent on material property and are determined from experimental data. Paris equation does not take into account the crack growth behaviour in threshold and unstable fracture regions. The other popularly used crack growth models implemented are Walker, Erdogan and Ratwani, Klesnil and Lucas, Forman and Forman New-de-Koning. The details of these models are presented by Rama Chandra Murthy et al. (2004b).

\section{Crack Growth Models for Variable Amplitude Loading (VAL)}

The effect of tensile overload has been reported by many investigators (Rama Chandra Murthy et al., 2004a). Single tensile overload introduce significant crack growth delay depending on the overload ratio (OLR). A superimposed single overload during CAL is the simplest case of VAL. The application of single overload will cause significant decrease in the crack growth rate for a large number of cycles subsequent to the overload. This phenomenon is referred to as crack retardation. Since analytical modeling of crack closure is very difficult, models based on yield zone concept are generally employed in the analytical investigation. It is well known that the widely used Wheeler and Generalised Willenborg residual stress models are based on yield zone concept assuming that crack growth retardation is caused by compressive residual stresses acting at the crack tip. In the present studies, Wheeler model has been employed to consider the retardation effects due to overload. Brief description about improved Wheeler model is given below (Ramachandra Murthy et al., 2004a).

\section{Improved Wheeler Residual Stress Model}

This model assumes that the crack growth retardation is caused by compressive residual stresses acting at the crack tip. Wheeler employs the residual stress retardation model to account for crack growth retardation due to tensile overload (Fig. 6). The development of Wheeler model begins with the basic crack growth equation 


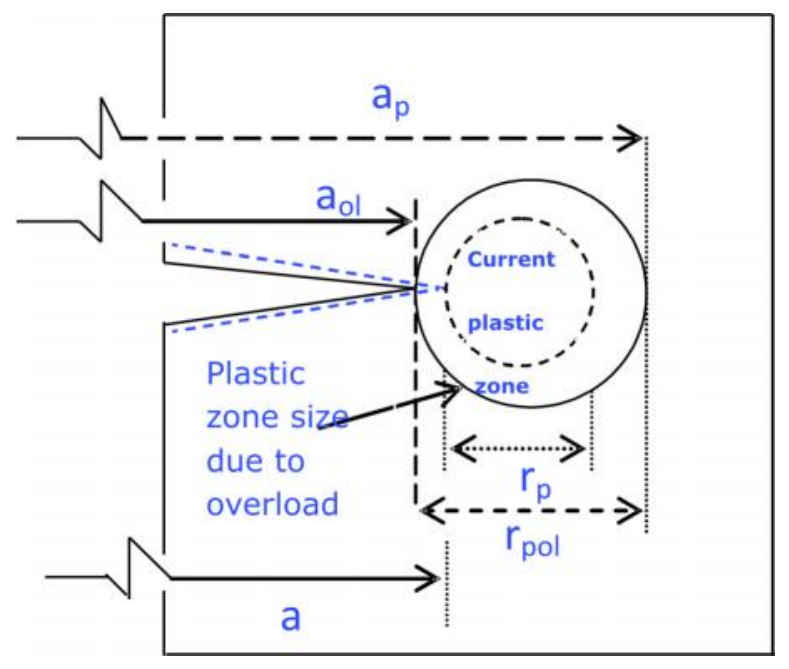

Fig. 6: Wheeler residual stress model

$$
\frac{d \mathrm{a}}{d N}=f(\Delta K)
$$

Since the load is discontinuous variable, the crack growth can be computed using cycle-by-cycle approach

$$
\mathrm{a}_{n}=\mathrm{a}_{o}+\sum_{i=1}^{N} f\left(\Delta K_{i}\right)
$$

where, $a_{n}=$ final crack length after $\mathrm{N}$ cycles, $a_{\mathrm{o}}=$ initial crack length $\Delta K_{i}=$ stress intensity factor range for cycle iTo account for crack growth retardation, Wheeler introduced a retardation parameter, $\mathrm{C}_{\mathrm{pi}}$, Eq. (19) then reduces to

$$
\mathrm{a}_{n}=\mathrm{a}_{o}+\sum C_{p i} f\left(\Delta K_{i}\right)
$$

The retardation parameter is calculated as shown below

$$
\begin{aligned}
C_{p i} & =\left(\frac{r_{p}}{\left(\mathrm{a}_{p}-\mathrm{a}\right)}\right)^{m_{1}} \text { for }\left(\mathrm{a}+r_{p}\right)<\mathrm{a}_{p} \\
& =1.0 \text { for }\left(\mathrm{a}+r_{p}\right)>\mathrm{a}_{p}
\end{aligned}
$$

where, $r_{p}=$ extent of current plastic zone $\left(\mathrm{a}_{p}-\mathrm{a}\right)=$ distance from crack tip to elastic-plastic interface (refer to Fig. 6) $m_{1}=$ shaping exponent, which is generally obtained through experiments. The value of $m_{1}$ depends on applied overload, crack size and width of the plate.

Expressions have been proposed for evaluation of shaping exponent $m_{1}$ for various geometries considering overload, crack size and width of the plate. Details can be found in Ramachandra Murthy et al. (2004a).

It is observed that $C_{p i}$ is minimum immediately after the application of overload, when $\left(a_{p}-a\right)$ has its maximum value. As 'a' approaches $a_{p}, C_{p i}$ increases.

\section{Remaining Life Prediction}

Remaining life for flat sheets/stiffened plates or tubular joints can be estimated using any of the crack growth models. The models give an empirical formula for da/ $\mathrm{dN}$ where $\mathrm{dN}$ is the increment in crack length and $\mathrm{dN}$ is the corresponding remaining life. To estimate the remaining life the following steps are followed:

1. The value of remaining life $(\mathrm{N})$ is initialized to zero at first.

2. The value of $K$ for each crack length $a_{i}$ starting with the initial crack length is calculated. The calculation uses the value of $\mathrm{a}_{\mathrm{avg}}\left(=\left(\mathrm{a}_{\mathrm{i}}+\mathrm{a}_{\mathrm{i}+1}\right) / 2\right)$ for each computation.

3. The value of SIF corresponding to each of the crack length is computed using the analytical expressions/interpolation method described earlier.

$$
\mathrm{K}=\mathrm{f}(\mathrm{a}) \sigma \sqrt{\pi \mathrm{a}}_{\mathrm{avg}}
$$

4. The value of $\Delta \mathrm{K}$ is calculated for crack growth models other than Walker using appropriate formula. In case of Walker model the value of $K_{\max }$ is calculated.

5. Apply the retardation model if needed.

6. The value of $\Delta \mathrm{K}$ or $\mathrm{K}_{\max }$ is passed to the function that calculates the value of $\mathrm{dN}$ provided $\Delta \mathrm{K}$ or $\mathrm{K}_{\max }$ is greater than the $\mathrm{K}_{\mathrm{th}}$ of the material. The function returns value of $\mathrm{dN}$. This value is added to the existing value of remaining life $(\mathrm{N})$. 


$$
\mathrm{dN}=\frac{\mathrm{da}}{\mathrm{C}(\mathrm{K})^{\mathrm{m}}}
$$

7. The values of crack length, SIF and $\mathrm{N}$ are written to a file for plotting graphs.

8. Steps 2 to 5 are repeated until the crack length becomes critical.

\section{Residual Strength Evaluation}

Residual strength (remaining strength under the presence of crack) can be computed by using

(i) plastic collapse condition or yield criterion

(ii) Fracture toughness criterion and

(iii) Remaining life approach.

First two approaches are well known. The third approach is proposed by authors (Ramachandra Murthy et al. 2009).

Brief description of residual strength evaluation using remaining life approach is presented below:

Irwin proposed the following stress intensity factor $\mathrm{K}_{\mathrm{s}}$ to quantify the intensity of the stress field surrounding the crack tip in a finite width plate with a remote stress, $\sigma$ :

$$
\mathrm{K}_{\mathrm{s}}=\beta \sigma \sqrt{\pi \mathrm{a}}
$$

where $\mathrm{a}=$ half-length of the crack, $\beta=$ Geometry factor

Hence such a plate with a half crack $a_{x}$, will fracture when the applied stress $\sigma_{x}$ satisfies the equation

$$
\mathrm{K}_{\mathrm{c}}=\beta \sigma_{x} \sqrt{\pi \mathrm{a}_{x}}
$$

where $\mathrm{K}_{\mathrm{c}}=$ critical SIF, which is a material property. The rate at which the crack grows under constant amplitude cyclic loading can be derived from the following equation that was proposed by Paris and Erdogan (1963).

$$
d \mathrm{a} / d N=C(\Delta K)^{m}
$$

which can be written in the following integral form to give the number of cycles $\mathrm{N}_{\mathrm{f}}$ that are required for a crack of initial length $2 \mathrm{a}_{\mathrm{i}}$ to propagate to a crack length $2 \mathrm{a}_{\mathrm{x}}$ :

$$
N_{f}=\int_{2 \mathrm{a}_{i}}^{2 \mathrm{a}_{x}} \frac{d \mathrm{a}}{C(\Delta K)^{m}}
$$

where $\mathrm{C}$ and $\mathrm{m}$ are Crack growth constants and $\Delta \mathrm{K}$ $=$ range of SIF by the cyclic load $\Delta \sigma$.

From Eq. (24)

$$
\Delta \mathrm{K}=\beta \Delta \sigma \sqrt{\pi \mathrm{a}}
$$

and from Eq. (25)

$$
\mathrm{a}_{\mathrm{x}}=K_{c}^{2} / \beta^{2} \sigma_{x}^{2} \pi
$$

Substituting Eqs. (28) and (29) into (26) and integrating gives the following residual strength curve, where $\sigma_{c}$ is the residual strength after $\mathrm{N}_{\mathrm{c}}$ cycles of load:

$$
N_{c}=D_{1}-S_{1}\left(1 / \sigma_{c}^{2}\right)^{(1-(m / 2))}
$$

where

$$
D_{1}=\left(2 a_{i}\right)^{1-(m / 2)} /\left[C \beta^{m}(\Delta \sigma)^{m} \pi^{\frac{m}{2}}\left(\frac{m}{2}-1\right)\right]
$$

and

$$
S_{1}=\left(\frac{2 K_{c}^{2}}{\beta^{2} \pi}\right)^{1-(m / 2)} /\left[C \beta^{m}(\Delta \sigma)^{m} \pi^{\frac{m}{2}}\left(\frac{m}{2}-1\right)\right]
$$

where for a fixed initial crack size $a_{i}$ the parameters $\mathrm{D}_{1}$ and $\mathrm{S}_{1}$ are constant.

The residual strength of a plate/panel is the least value obtained by using the above three criterions.

In general, the construction of a residual strength diagram involves three major steps:

(a) The development of the relationship between the applied stress $\sigma$, the crack length parameter ' $\mathrm{a}$ ', and the applied stress-intensity factor ' $\mathrm{K}$ ' for the given structural configuration. 
(b) The selection of an appropriate failure criterion based on the expected material behavior at the crack tip

(c) The fracture strength $\left(\sigma_{\mathrm{f}}\right)$ values for critical crack sizes $\left(a_{c}\right)$ are obtained by utilizing the results of the first two steps and residual strength diagram $\left(\sigma_{\mathrm{f}}\right.$ vs. $\left.\mathrm{a}_{\mathrm{c}}\right)$ for the given structural configuration is plotted.

\section{Numerical Studies}

Example 1: Fracture Analysis of a Rectangular Plate with Center Crack under Uniaxial Tension

Fracture analysis of a rectangular plate with center crack subjected to uniaxial tensile loading (mode I) as shown in Fig. 7 has been conducted to compute SERR and SIF at the crack tip. One quarter of the plate with symmetric boundary conditions has been idealized. FE idealization of the plate using 4-noded element is shown Fig. 8. Basic stress analysis of the plate has been carried out by employing 4-noded, 8noded (regular and quarter-point), 9-noded and 12noded finite elements. SERR has been evaluated by using NI-MVCCI technique. For evaluating the integrals associated with NI-MVCCI technique, Gauss integration technique has been used with rules of 2, 3 and 4 for 4-noded, 8-noded/9-noded and 12noded elements respectively, while for 8-noded QPE different rules have been employed. Plane strain conditions have been assumed at the crack tip to compute SIF by using SERR value obtained using NI-MVCCI technique. The variation of SIF with respect to $\Delta \mathrm{a} / \mathrm{a}$ and W/a is shown in Fig. 9 along with the results obtained by using MVCCI technique (closed form equations) and the finite plate solution of Rooke and Cartwright (1976).

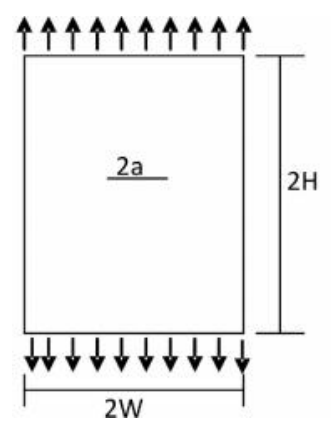

Young's Modulus, $E=10000.0 \mathrm{~N} / \mathrm{mm}^{2}$
Poison's ratio, $v=0.0$
Thickness, $\mathrm{t}=10.0 \mathrm{~mm}$
$\mathrm{H}=250 \mathrm{~mm}$
$W=100 \mathrm{~mm}$
$a=20 \mathrm{~mm}$

Fig. 7: Rectangular plate with centre under uniaxial tension

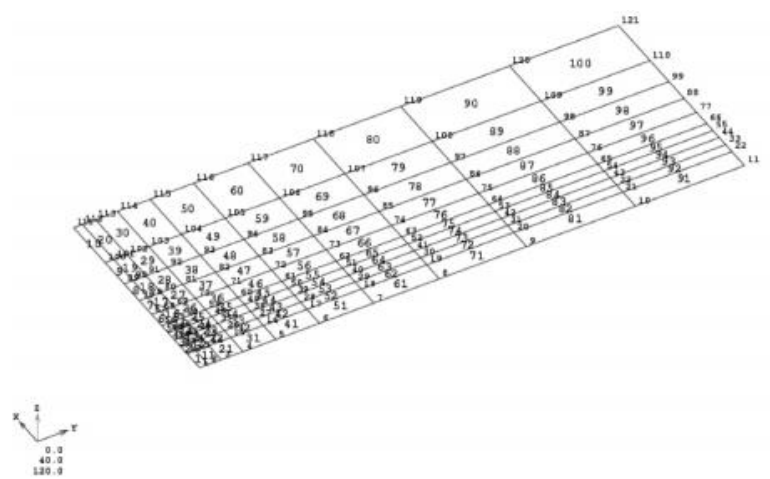

Fig. 8: FE idealization of rectangular plate (quarter symmetry) (a) Variation w.r. to $\Delta \mathrm{a} / \mathrm{a}$ (b) Variation w.r. to $\mathbf{W} / \mathbf{a}$
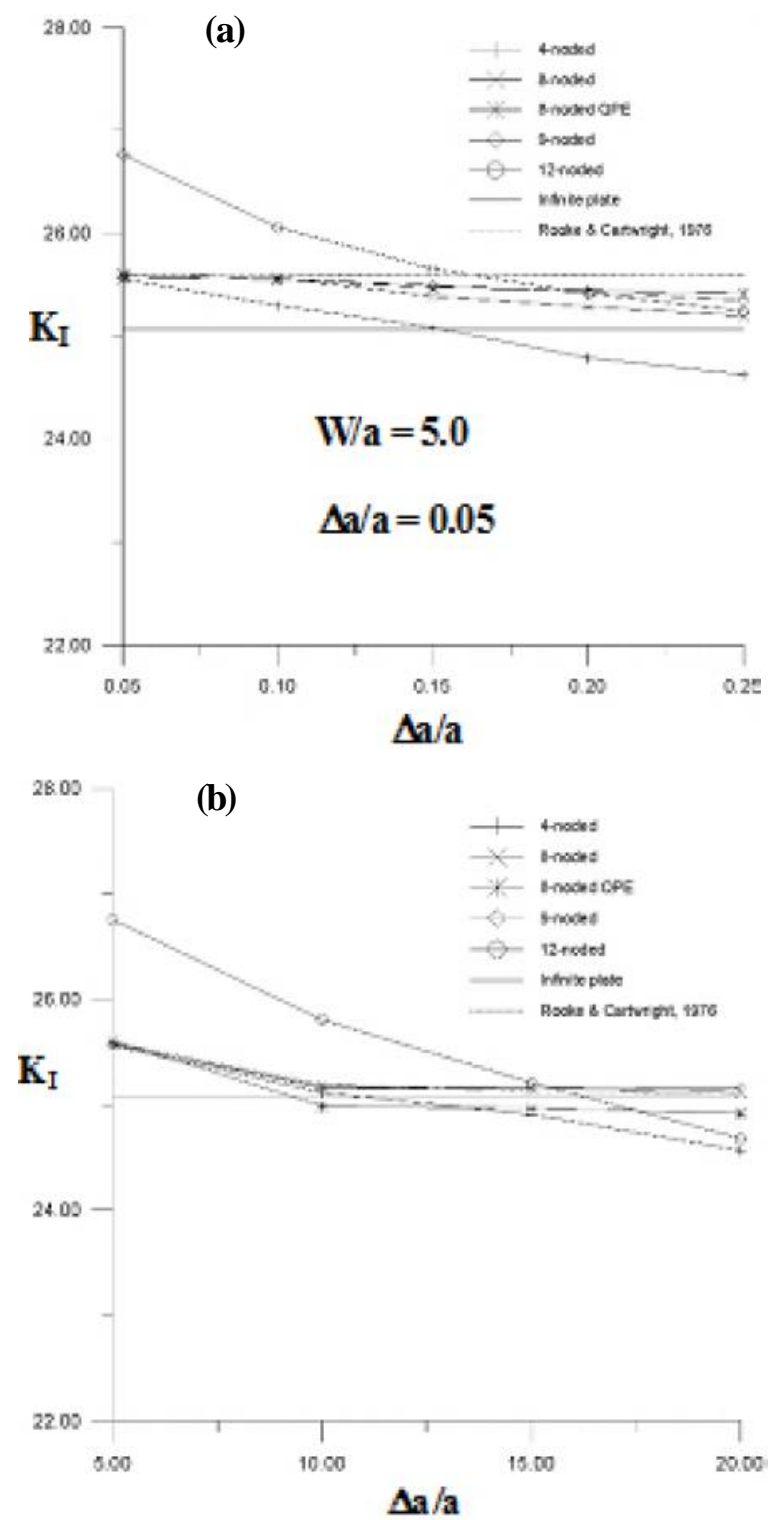

Fig. 9: Variation of SIF for rectangular plate with center crack (Mode I) 


\section{Example 2: Damage tolerant curves generation for plate with a centre crack using XFEM}

A $350 \mathrm{WT}$ steel plate of width (2b) $100 \mathrm{~mm}$, height (2h) $310 \mathrm{~mm}$ with a centre crack is subjected to tensile fatigue loading. The details of the problem are given below:

- $\quad$ Size of panel $2 \mathrm{~h} \times 2 \mathrm{~b}=310 \times 100 \mathrm{~mm}$

- Material is homogeneous, isotropic and made up of 350WT Steel

- Young's modulus $=2 \times 10^{5} \mathrm{~N} / \mathrm{mm}^{2}$

- $\quad$ Poisson's ratio $=0.3$

- Panel is modelled with four node bilinear element of size $2 \mathrm{~mm} \times 2 \mathrm{~mm}$

- Stiffener is modelled with two node truss element

- Plane strain linear elastic fracture mechanics assumption

- $\quad$ Paris crack growth law constants $\mathrm{C}=1.02 \mathrm{x}$ $10^{-11}, \mathrm{~m}=2.94$

- $\quad$ Fracture toughness $\mathrm{K}_{\mathrm{IC}}=50 \mathrm{MPa} \sqrt{m}$

The objective is to predict the number of fatigue cycles required for given applied stress range for a plate with centre crack using XFEM and get the applied stress range versus number of fatigue cycles required to fail i.e., the damage tolerant curves. The failure criterion of the plate has been set as the crack length reaching $60 \%$ of plate width or fracture toughness of the plate whichever is earlier. Stress range is varied from 200MPa to 40MPa for various initial crack length to plate width ratios (a/b) $0.1,0.2$ and 0.3. For each stress range XFEM analysis is carried out as per Flow chart of Fig. 6 to find the number of fatigue cycles required to fail. The results obtained are shown in Fig. 10, as applied stress range versus number of fatigue life cycles in the form of semi-log plot. From the results shown in Fig. 10, as the external applied stress is decreasing number of fatigue life cycles taken by the component is increasing. This figure gives the quick idea about the number of cycles a crack of initial length to plate width ratio between 0.1 and 0.3 can take for applied stress range between 200 and 40MPa.

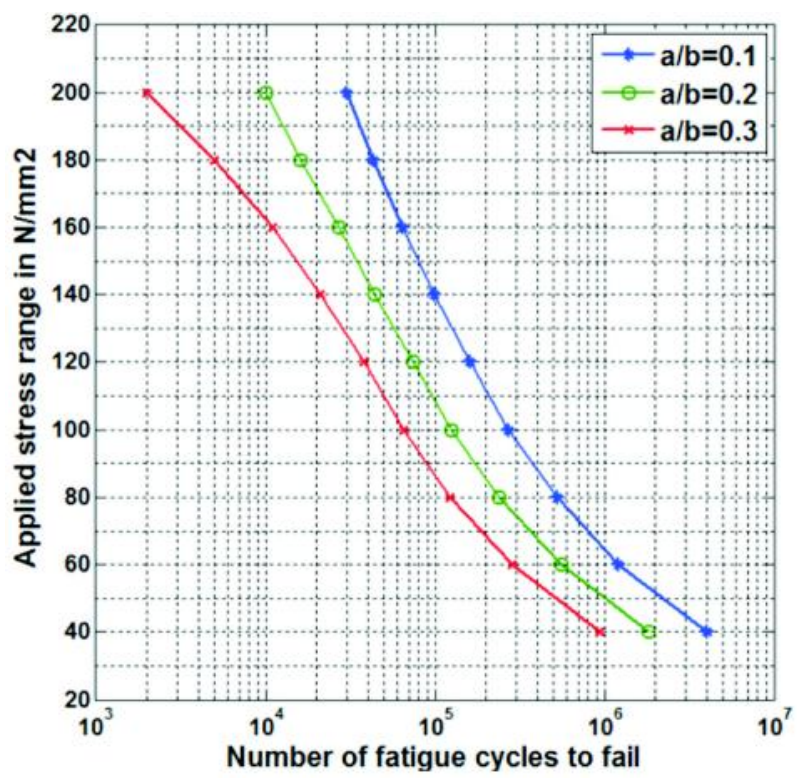

Fig. 10: Damage tolerant curves for plate with centre crack

\section{Example 3: Remaining life Prediction and Residual strength evaluation of 350WT Steel}

Crack growth studies, remaining life prediction and residual strength evaluation has been carried out for plate with a centre crack with stiffeners. The plate is made up of $350 \mathrm{WT}$ steel and the details of the problem are same as discussed earlier. The loading spectrum and occurrence of overload is shown in Fig. 11.

Fig. 12 shows the variation of life for different stiffener areas (as non-dimensional parameter) under CAL and VAL (OLR=1.25, 1.5 and 1.75). From Fig. 12 , it can be observed that

The predicted remaining life of stiffened panel under CAL increases with increase of stiffener area and is about $209 \%$ higher for stiffener area of $100 \mathrm{~mm}^{2}$ as compared to the corresponding unstiffened case.

$>\quad$ The predicted remaining life of stiffened panel under VAL with OLR $=1.25,1.5,1.75$ and for different OLs increases with increase of stiffener area and is about $237 \%$ higher for stiffener area of $100 \mathrm{~mm}^{2}$ with OLR $=1.75$ and no. of OLs $=$ 2 compared to the respective unstiffened case.

$>$ The percentage increase in remaining life of stiffened panel compared unstiffened plate under 


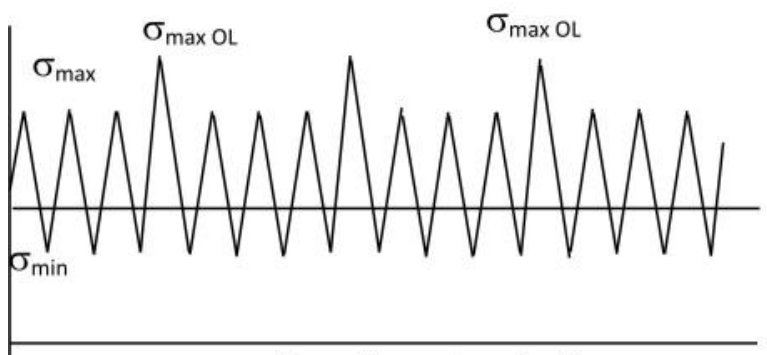

No. of overloads: 3

Crack length at occurrence of overload $=15,20,25 \mathrm{~mm}$

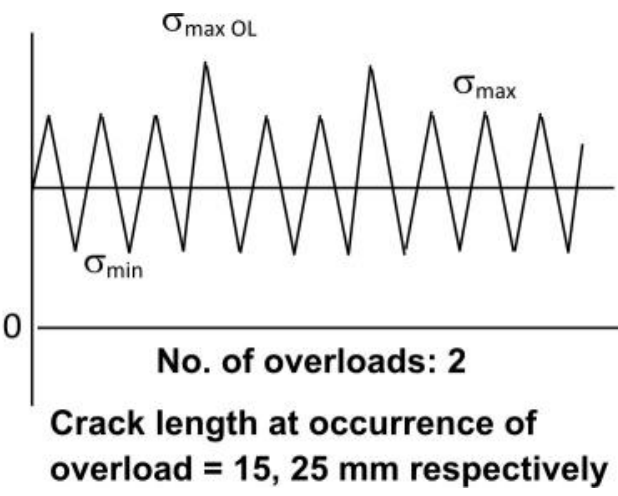

Fig. 11: Loading Spectrum

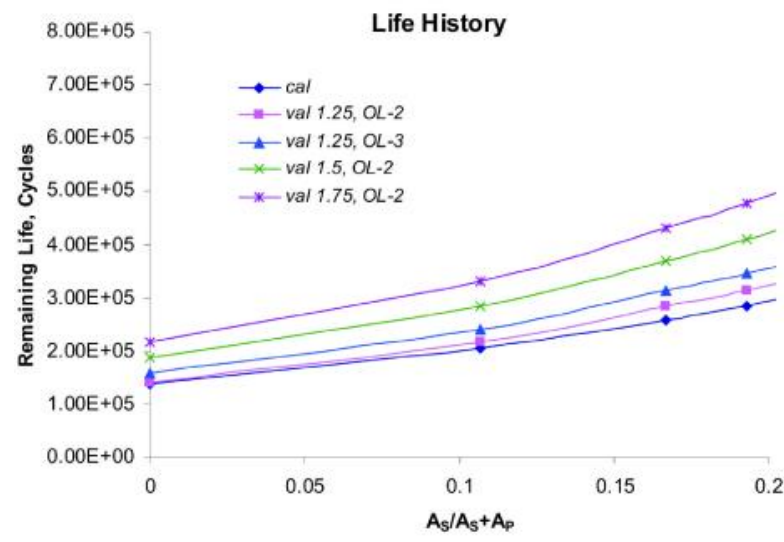

Fig. 12: Remaining life for different stiffener sizes under CAL and VAL

VAL is approximately same for particular stiffener area and overload ratio and no. of OLs.

$>$ Remaining life is influenced by the number of overloads and occurrence of overload.

Fig. 13 shows the plot of crack length vs residual strength predicted using plastic collapse condition (yield condition), fracture toughness criterion and remaining life approach. It can be observed from Fig. 13 that the residual strength predicted using remaining life approach are lower compared to those values predicted by using other two approaches and will govern the design.

\section{Concluding Remarks}

This paper presents an overview of the advanced methodologies for fracture analysis and damage tolerant evaluation of structural components. The novel contributions presented in this paper include:

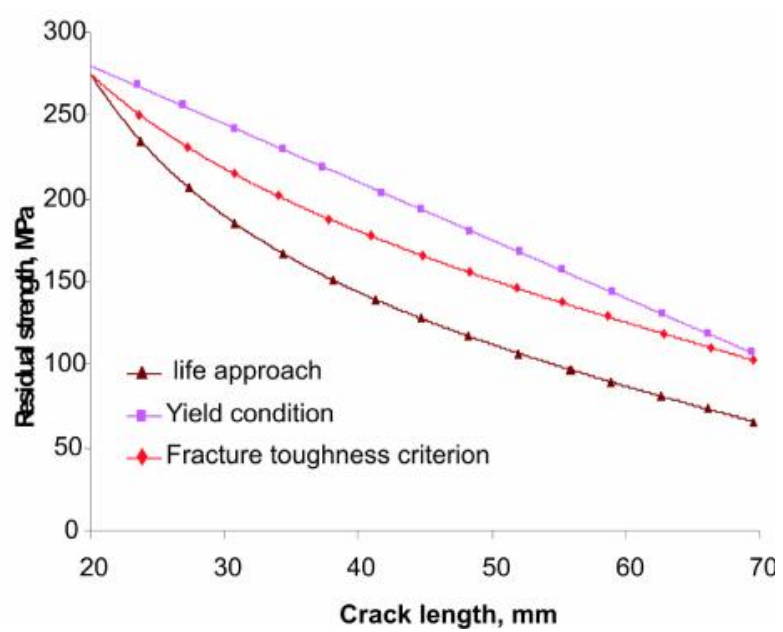

Fig. 13: Crack length vs residual strength

- $\quad$ NI-MVCCI technique for fracture analysis

- $\quad$ XFEM for stiffened panels

- Improved Wheeler residual stress model to account for retardation effects due to overload

- Residual strength evaluation using remaining life approach

NI-MVCCI technique has been demonstrated for 4-noded bilinear, 8-noded Serendipity (regular \& quarter-point), 9-noded Lagrangian and 12-noded cubic isoparametric finite elements. An overview on XFEM for fracture analysis of structural components has been presented. Methodologies for crack growth and remaining life assessment of structural components under constant and variable amplitude loading have been presented. Brief details related to evaluation of residual strength has been highlighted. Numerical studies on fracture analysis to compute SERR and 
SIF and remaining life assessment have been conducted and the results are compared with the respective analytical/experimental results. From the studies, it is noted that the fracture parameters computed are in good agreement with those of literature values. Damage tolerant curves in the form of S-N curves have been developed for various stress ranges. These curves are very much useful for design of the structural components under fatigue loading. Crack growth analysis and remaining life prediction has been carried out for stiffened plate with centre crack under constant and variable amplitude loading. The residual strength has been evaluated by using

\section{References}

Belytschko T and Black T (1999) Elastic crack growth in finite elements with minimal remeshing International Journal for Numerical Methods in Engineering 45 601-620

Broek D (1982) Elementary engineering fracture mechanics Martinus Nijhoff Publications The Hague

Chu H P Hauser J A and Sikoram J P (1982) Fatigue crack growth in stiffened panels under pressure loading-design of fatigue and fracture resistant structures ASTM STP $\mathbf{7 6 1}$ 345-72

Dexter R J and Pilarski P J (2002) Crack propagation in welded stiffened panels Journal of Constructional Steel Research 58 1081-1102

Dolbow J Moes N and Belytschko T (2000) Discontinuous enrichment in finite elements with a partition of unity method Finite Elements in Analysis and Design 36 235260

Ghassem M M and Rich T P (1983) The fracture diagram: a new design tool for stiffened panels The American Institute of Aeronautics and Astronautics Journal 21 1294-1300

Guoshou Liu Shiyue Wang and Xijie Yang (2012) A Simple numerical simulation of crack growth rate Procedia Engineering 31 557-562

Mahmoud H N and Dexter R J (2005) Propagation rate of large cracks in stiffened panels under tension loading Marine Structures 18 265-288

Melenk J M and Babuska I (1996) The partition of unity finite element method: basic theory and applications Computer Methods in Applied Mechanics and Engineering 139 289314

Moes N Dolbow J and Belytschko T (1999) A finite element method for crack growth without remeshing International Journal for Numerical Methods in Engineering 46131 150 various approaches and found that the residual strength predicted by remaining life approach governs the design. It can be observed that the size of the stiffener, initial crack length, magnitude of overload and number of overloads play significant role for the prediction of remaining life and residual strength of stiffened panels.

\section{Acknowledgements}

The authors acknowledge fruitful discussions and contributions of Ms. Smitha Gopinath and Mr M. R. Nanda Kumar related to work on XFEM.

Murakami Y (1988) Stress intensity factors handbook Pergamon Press Oxford

Nanda Kumar M R Ramachandra Murthy A Smitha Gopinath and Nagesh R Iyer (2016) XFEM for fatigue and fracture analysis of cracked stiffened panels Structural Engineering and Mechanics An Int'l Journal 57 65-89

Palani G S Dattaguru B and Iyer N R (2004) Numerically integrated modified virtual crack closure integral technique for 2-D crack problems Structural Engineering and Mechanics 18 731-744

Paris P and Erdogan F (1963) A critical analysis of crack propogation laws Journal of Basic Engineering 85 528533

Poe Jr C C (1971) Fatigue propagation in stiffened panels ASTM STP 486 79-98

Ramachandra Murthy A Palani G S and Nagesh R Iyer (2007) Remaining life prediction of cracked stiffened panels under constant and variable amplitude loading International Journal of Fatigue 29 1125-1139

Ramachandra Murthy A Palani G S and Nagesh R Iyer (2004a) An improved wheeler residual stress model for remaining life assessment of cracked plate panels CMC-Computers Materials and Continua 1289-300

Ramachandra Murthy A Palani G S and Nagesh R Iyer (2004b) State-of-art review on fatigue crack growth analysis under variable amplitude loading Institution of Engineers (Civil Engineering Division) 85 118-129

Ramachandra Murthy A Palani G S and Nagesh R Iyer (2009) Residual Strength Evaluation of Unstiffened and Stiffened Panels under Fatigue Loading Structural Durability and Health Monitoring 5 201-226 
Rooke D P and Cartwright D J (1976) Compendium of stress intensity factors Her Majesty's Stationary Office London

Saves C S T Germes Davy A and Barrau J J (2001) Prediction of the longitudinal crack behaviour of stiffened curved panels International Journal of Fatigue 23 147-158

Schijve J (2003) Fatigue of structures and materials in the 20th century and the state of the art International Journal of Fatigue 25 679-702

Taheri F Trask D and Pegg N (2003) Experimental and analytical investigation of fatigue characteristics of $350 \mathrm{WT}$ steel under constant and variable amplitude loadings Marine Structures 16 69-91

Tong P and Pian T H (1973) On the convergence of the finite element method for problems with singularity International Journal of Solids and Structures 9 313-321
Vasudevan A K Sadananda K and Glinka G (2001) Critical parameters for fatigue damage International Journal of Fatigue 23 S39-S53

Wen P H Aliabadi M H and Young A (2000) Stiffened cracked plates analysis by dual boundary element method International Journal of Fracture 106 245-258

Wen P H Aliabad M H and Young A (2003) Fracture mechanics analysis of curved stiffened panels using BEM International Journal of Solids and Structures 40 219-236

Zheng X (2001) On some basic problems of fatigue research in engineering Inte4rnational Journal of Fatigue 23 751-766

Zienkiewicz O C and Taylor R L (2000) The Finite element method - Vol. I: The basis \& Vol. II: Solid mechanics ButterworthHienemann Ltd. 